\title{
Psycho-acoustic evaluation of the indoor noise in cabins of a naval vessel using a back-propagation neural network algorithm
}

\author{
Hyung-Suk Han \\ Naval System Research Team, Busan Center, Defense Agency for Technology and Quality, Busan, Korea
}

\begin{abstract}
The indoor noise of a ship is usually determined using the A-weighted sound pressure level. However, in order to better understand this phenomenon, evaluation parameters that more accurately reflect the human sense of hearing are required. To find the level of the satisfaction index of the noise inside a naval vessel such as "Loudness" and "Annoyance", psycho-acoustic evaluation of various sound recordings from the naval vessel was performed in a laboratory. The objective of this paper is to develop a single index of "Loudness" and "Annoyance" for noise inside a naval vessel according to a psycho-acoustic evaluation by using psychological responses such as Noise Rating (NR), Noise Criterion (NC), Room Criterion (RC), Preferred Speech Interference Level (PSIL) and loudness level. Additionally, in order to determine a single index of satisfaction for noise such as "Loudness" and "Annoyance", with respect to a human's sense of hearing, a back-propagation neural network is applied.
\end{abstract}

KEY WORDS: Noise in a naval vessel; Indoor noise; Psycho-acoustic evaluation; Linear regression; Back-propagation neural network.

\section{INTRODUCTION}

Even though the concept of noise and vibration in the industry is primarily focused on the reliability of machinery, there has been a shift in research focus to examining the ways in which convenience and pleasantness of noise and vibration relates to a human's level of comfort. Low noise inside a ship or offshore structure is necessary to create a comfortable internal environment. In the past, ships were regarded as very loud spaces. However, environmental noise is one of the important properties of a ship because employees in the cabin and office areas of a ship require a low noise environment. Additionally, when considering a naval vessel, where crews live for a longer period of time compared to crews on a merchant ship, the reduction of indoor noise is particularly important.

Crews that live for an extended period of time in offshore structures require a quiet space for rest. The appropriate level of noise inside the cabin of an offshore structure, such as a floating production, storage and offloading (FPSO) structure, is $45 \mathrm{dBA}$ (Joo and Lee, 2009). When compared to the IMO's standard for noise levels in the cabin of a merchant ship ( $60 \mathrm{dBA}$ max.), this is very low (IMO, 1982). The standard for a naval vessel is similar to an FPSO and requires a more conservative level of noise. Since the noise limit suggested by IMO is not proper to apply to the naval vessel, the new specification of the noise level in the naval vessel which can respect crew's satisfaction for the noise is necessary.

Even though specifications of indoor noise have been suggested by various research institutes, much of the research related to the noise limit is still being performed because the specifications cannot perfectly replicate a human's sense of hearing. Ju et al. (2010) researched noise produced by heavy construction machines using psycho-acoustic evaluation. He suggested

Corresponding author: Hyung-Suk Han

e-mail:daerihan@hanmail.net 
the limit of the noise for these machines by completing a correlation analysis that examined the measured noise level and a human's perception of that noise. Chun et al. (2008) performed psycho-acoustic evaluation on the annoyance of noise resulting from express trains and local trains and suggested that the noise limit should be different according to the type of train. He suggested the method to make a limit of the noise from the train by the psycho-acoustic evaluation in a laboratory. Lee et al. (2008) suggested the relationship between subjective response by human and the noise from water supply and drain installation in the apartment bathroom. In this paper, the noise quality could be evaluated with psycho-acoustic evaluation and the relationship between the noise and human's response with sound parameters such as $d B A, \mathrm{NR}$ and $\mathrm{N}$.

Tamura et al. (1997) researched experimentally about the effect on sleep from the noise in a ship based on the IMO standard. The psycho-acoustic evaluation was performed in a laboratory with the audio tracks recorded in various ships. He found that the noise over $65 d B A$ can cause sleeping problem for a human. Lee et al. (2010) researched about the relationship between environmental noise and sleeping problem of a human. He performed the psycho-acoustic evaluation for 2 weeks with 20 people. Through the statistical analysis, it can be found that the loudness of the noise affect the sleeping problem rather than quality or pattern of the sound.

Recently, researchers have attempted to replicate human behavior using artificial intelligence. A neural network is one of the popular artificial intelligence techniques because it can be used to represent a non-linear response, such as satisfaction regarding indoor noise. Nannariello et al. (2001) used the neural network algorithm to predict appropriate speech levels in university classrooms. His research examined the relationship between sound propagation levels and design factors of the classrooms that affect sound propagation, such as size, listener location, source location, and reverberation time. For the booming sound of a vehicle, Lee et al. (2006) suggested an evaluation algorithm that embodied a back-propagation neural network that used general sound quality parameters, such as loudness, sharpness, roughness, and fluctuation strength.

In this research, a psycho-acoustic evaluation is performed to determine the satisfaction index of the noise inside a naval vessel such as "Loudness" and "Annoyance" using recorded audio tracks. Audio tracks were recorded in various naval vessels and created by modulation with recorded audio tracks. Through psycho-acoustic evaluation, the limit of the satisfaction index of the noise inside a naval vessel was determined using various parameters that evaluated noise, such as Aweighted sound pressure level, NR, NC, RC, PSIL, and Zwicker's Loudness level. Additionally, the linear regression and back-propagation neural network algorithm were applied to determine the satisfaction index of the noise using various evaluation parameters.

\section{METHODOLOGY}

In previous research, a crew living in a naval vessel completed a survey to determine their satisfaction with the level of noise (Han et al., 2010). The result of this survey showed that the satisfaction index of the noise could be determined using a multiple linear regression model. However, the maximum allowable noise level included some errors because the survey was completed by numerous crews living in different cabins on different ships. Since the conditions of the ship were not uniform, the sounds that were measured could not be precisely matched with the surveyed results.

Therefore, in order to reduce errors in the survey results, the psycho-acoustic evaluation was performed at the same place and the recorded sound sources were assessed by a finite number of evaluators. In our previous research (Hong et al., 2010), the psycho-acoustic evaluation was performed with recorded and modulated audio tracks. Using a B\&K type 4189 microphone and a Type 3560B data acquisition system, these audio tracks were recorded in various cabins on the naval vessel. Psycho-acoustic evaluation was performed in a small auditorium as shown in Fig. 1 (Hong et al., 2010). Ten jurors evaluated the 50 different audio tracks. The background noise was $30 d B A$, sufficiently low compared to the replaying audio tracks $(45 d B A \sim 75 d B A)$. The jury consisted of 8 men and 2 women, aged 30 to 50, who worked for the DTaQ (Defense agency for Technology and Quality).

Replayed audio tracks were evaluated using an index level ("Loudness" and "Annoyance"), which was derived from the survey shown in Table 1. The average "Loudness" and "Annoyance" levels for each audio track were calculated based on the 10 jurors' response to the survey shown in Table 1 (Hong et al., 2010). 


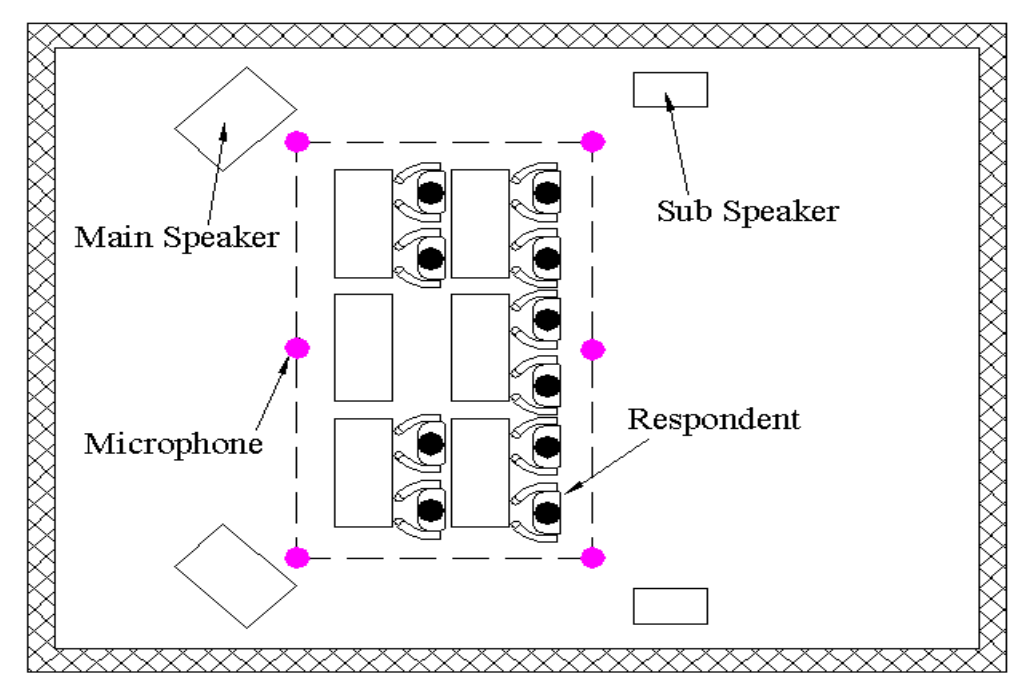

(a) Schematic diagram of the psycho-acoustic evaluation.

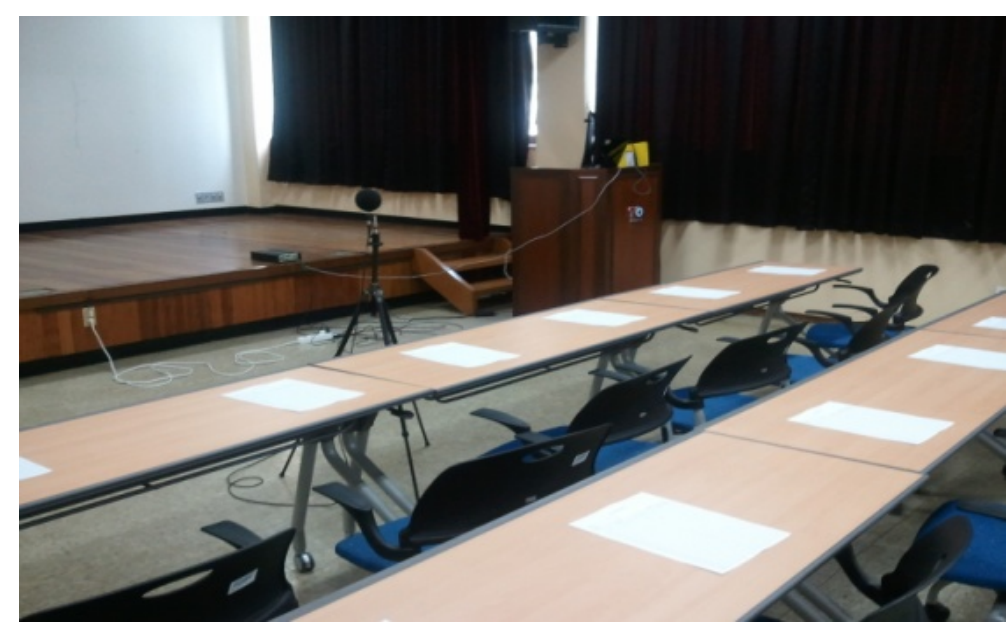

(b) Picture of the room for the psycho-acoustic evaluation.

Fig. 1 Set-up of the psycho-acoustic evaluation.

Table 1 Survey questions regarding the expression of the index level $\left(\mathrm{L}_{\mathrm{i}}\right)$.

\begin{tabular}{|c|c|c|c|c|c|c|}
\hline \multirow{2}{*}{$\mathrm{i}$} & \multicolumn{2}{|c|}{ Question } & \multicolumn{5}{|c|}{ Index level $\left(\mathrm{L}_{\mathrm{i}}\right)$} \\
\cline { 3 - 6 } & & 5.0 & 4.0 & 3.0 & 2.0 & 1.0 \\
\hline 1 & How loud is the given sound? & Very loud & Loud & Normal & Quiet & Very quiet \\
\hline 2 & $\begin{array}{c}\text { How annoying is the given sound? } \\
\text { (Annoyance) }\end{array}$ & Very Annoyed & Annoyed & Normal & Not Annoyed & Convenient \\
\hline
\end{tabular}

When the psycho-acoustic evaluation was performed, sound parameters, such as A-weighted sound pressure level, NC, NR, RC, PSIL, and Zwicker's Loudness, were measured simultaneously. NC, NR, and Zwicker's Loudness are evaluation parameters for indoor noise that are based on equal loudness contour; they are widely used to evaluate room acoustics. NC (Beranek, 1957) were developed by Beranek and established in U.S. for rating indoor noise, noise from air-conditioning equipment etc. The method consists of a set of criteria curves extending from 63 to $8000 \mathrm{~Hz}$, and a tangency rating procedure. The criteria curves define the limits of octave band spectra that must not be exceeded to meet occupant acceptance in certain spaces. The NC rating can be obtained by plotting the octave band levels for a given noise spectrum. The noise spec- 
trum is specified as having a NC rating same as the lowest NC curve which is not exceeded by the spectrum. In Europe it is common to use NR (Kostan and OS, 1962). NR curves were developed by the International Organization for Standardization (ISO) to determine the acceptable indoor environment for hearing preservation, speech communication and annoyance.

In describing the subjective loudness of the sound, it is necessary to consider the characteristics of a human ear. This concept of the loudness is loudness level. The loudness level of a sound is determined by subjective comparison of the loudness of the sound to that of a $1000 \mathrm{~Hz}$ pure tone. The level measured phone is equal numerically to the sound pressure level, in $\mathrm{dB}$, of $1000 \mathrm{~Hz}$ tone, which deemed to be of equal loudness. In this paper, the loudness level is calculated by Zwicker's method (ISO, 1975).

$\mathrm{RC}$ curves for evaluating noise in a room or for specifications were built around the point of view that sound levels lower than those shown by the NC curve should always be specified for the bottom three-octave frequency bands ( 31.5 , 63 and $125 \mathrm{~Hz}$ ). The primary reason given is that if there are strong fluctuations or surging in the HVAC noise, the specified low-frequency octave-band levels for the RC curves ought to be low enough so that the noise is not disturbing. Even though RC was revised to RC Mark-II (Blaizer, 1997), original RC (Blaizer, 1981) is used in this paper. In addition, PSIL (ANSI, 1977) should be used for evaluations in a naval vessel because speech interference is an important potential hindrance when a command is given.

After the evaluation results of the 50 audio tracks, a simple linear regression was performed with an evaluated index level ("Loudness" and "Annoyance") and sound parameters (A-weighted sound pressure level, NR, NC, RC, PSIL and Zwicker's Loudness). The results determined that the R-square levels between them were very high, as is shown in Table 2 (Hong et al., 2010).

Table 2 Measured data and their R-square level.

\begin{tabular}{|c|c|c|c|c|c|c|c|}
\hline \multirow{2}{*}{ Items } & \multirow{2}{*}{ Max. } & \multirow{2}{*}{ Min. } & \multirow{2}{*}{ Average } & \multicolumn{2}{|c|}{ R-square level } & \multicolumn{2}{|c|}{$p$ value for analysis of variance } \\
\cline { 5 - 8 } & & & & Loudness & Annoyance & Loudness & Annoyance \\
\hline SPL $[d B A]$ & 72.0 & 48.1 & 59.5 & 0.893 & 0.834 & $<0.001$ & $<0.001$ \\
\hline NR & 69.4 & 43.9 & 55.9 & 0.873 & 0.829 & $<0.001$ & $<0.001$ \\
\hline NC & 71.4 & 41.1 & 56.2 & 0.849 & 0.825 & $<0.001$ & $<0.001$ \\
\hline RC & 68.1 & 47.1 & 57.0 & 0.831 & 0.758 & $<0.001$ & $<0.001$ \\
\hline PSIL & 64.3 & 40.4 & 52.5 & 0.883 & 0.800 & $<0.001$ & $<0.001$ \\
\hline Loudness level $[p h o n]$ & 88.2 & 67.6 & 77.6 & 0.929 & 0.866 & $<0.001$ & $<0.001$ \\
\hline
\end{tabular}

* The linear regression model is statistically significant when " $\mathrm{p}<0.05$ ".

According to a simple linear regression analysis, the parameters used in this research to evaluate sound quality in a naval vessel were sufficiently affected by the satisfaction indices for the noise, such as "Loudness" and "Annoyance".

However, there were some limitations to perform this psycho-acoustic evaluation such as below.

(1) The audiograms of jurors are different.

(2) The sample size is insufficient $(\mathrm{N}=10)$.

(3) The jurors are nor work or live in the naval vessel even though they knew the noise in a naval vessel very well.

\section{ANALYSIS}

\section{Multiple linear regression}

If the number of input variables is not a unit, the estimation of the relationship between the input and the output variable is performed by multiple linear regressions. There are multiple input variables derived from the satisfaction index for the noise, 
such as "Loudness" and "Annoyance". Multiple linear regressions were performed using A-weighted sound pressure level, NR, $\mathrm{NC}, \mathrm{RC}$, PSIL, and Loudness. A multiple linear regression model, having $p$ independent variables for $n$ data, can be determined using Eq. (1).

$$
\begin{gathered}
y=x \beta+\varepsilon \\
y=\left[\begin{array}{c}
y_{1} \\
y_{2} \\
\vdots \\
y_{n}
\end{array}\right], \quad x=\left[\begin{array}{ccccc}
1 & x_{11} & x_{21} & \cdots & x_{p 1} \\
1 & x_{12} & x_{22} & \cdots & x_{p 2} \\
\vdots & \vdots & \vdots & \cdots & \vdots \\
1 & x_{1 n} & x_{2 n} & \cdots & x_{p n}
\end{array}\right], \beta=\left[\begin{array}{c}
\beta_{1} \\
\beta_{2} \\
\vdots \\
\beta_{n}
\end{array}\right], \varepsilon=\left[\begin{array}{c}
\varepsilon_{1} \\
\varepsilon_{2} \\
\vdots \\
\varepsilon_{n}
\end{array}\right]
\end{gathered}
$$

where $y$ is the matrix of the dependent variable, $x$ is the matrix of the independent variable, $\beta$ is the matrix of the regression coefficient, and $\varepsilon$ is the matrix of the random error.

In Eq. (1), the least square $\operatorname{sum}(S)$ that is used to estimate the coefficients of the regression equation is given in Eq. (2).

$$
S=\sum_{i=1}^{n} \varepsilon_{i}^{2}=\varepsilon^{T} \varepsilon=(y-x \beta)^{T}(y-x \beta)=y^{T} y-2 \beta^{T} x^{T} y+\beta^{T} x^{T} x \beta
$$

When the partial differential is performed for Eq. (2) by $\beta$ and equal to 0, Eq. (3) can be derived. When $\beta$ satisfying with Eq. (3) is set to $\hat{\beta}$, Eq. (3) can be represented as is shown in Eq. (4).

$$
\begin{gathered}
\frac{\partial s}{\partial \beta}=-2 x^{T} y+2 x^{T} x \beta=0 \\
x^{T} \hat{\beta} x=x^{T} y
\end{gathered}
$$

Through Eq. (4), the coefficient of the regression equation can be calculated as is shown in Eq. (5), if the inverse matrix of $x^{T} x$ exists.

$$
\hat{\beta}=\left(x^{T} x\right)^{-1} x^{T} y
$$

When the multiple regression is performed in our previous research (Hong et al., 2010), the independent variables are Aweighted sound pressure level, NR, NC, RC, PSIL, and Zwicker's Loudness; the dependent variables are "Loudness" and "Annoyance"; and the regression equation is shown in Eq. (6) (7).

$$
\begin{aligned}
& L_{\text {Loudness }}=-18.3-0.367 d B A-0.0216 N R+0.0546 N C-0.452 R C+0.0039 P S I L+0.558 \text { Loudness } \\
& L_{\text {Annoyance }}=-13.4-0.31 d B A+0.0411 N R+0.0536 N C-0.0666 R C-0.0495 P S I L+0.469 \text { Loudness }
\end{aligned}
$$

The multiple regression analysis found that the R-square between "Loudness" and "Annoyance" and measured sound parameters were 0.954 and 0.907 respectively. The reason why "Annoyance" has a lower R-square level with respect to the measured sound parameters than "Loudness" is that "Annoyance" may be more subjective than "Loudness".

Fig. 2 shows the index level from the derived regression equation (Eq. (6) (7)) for 50 audio tracks that were used in the psycho-acoustic evaluation. Fig. 2 shows that the error is over $5 \%$ for the $L_{\text {linear }} / L_{\text {psycho }}$ curve, even though the estimation results from the multiple regression analysis coincides with the results from the psycho-acoustic evaluation. Here, $L_{\text {linear }}$ is 
the estimated satisfaction level ("Loudness" or "Annoyance") from the linear regression analysis and $L_{p s y c h o}$ is estimated from the psycho-acoustic evaluation. Therefore, in this research, a back-propagation neural network algorithm, one of the representative algorithms of artificial interference, is applied in order to reduce this error. This process will be described in the next section.

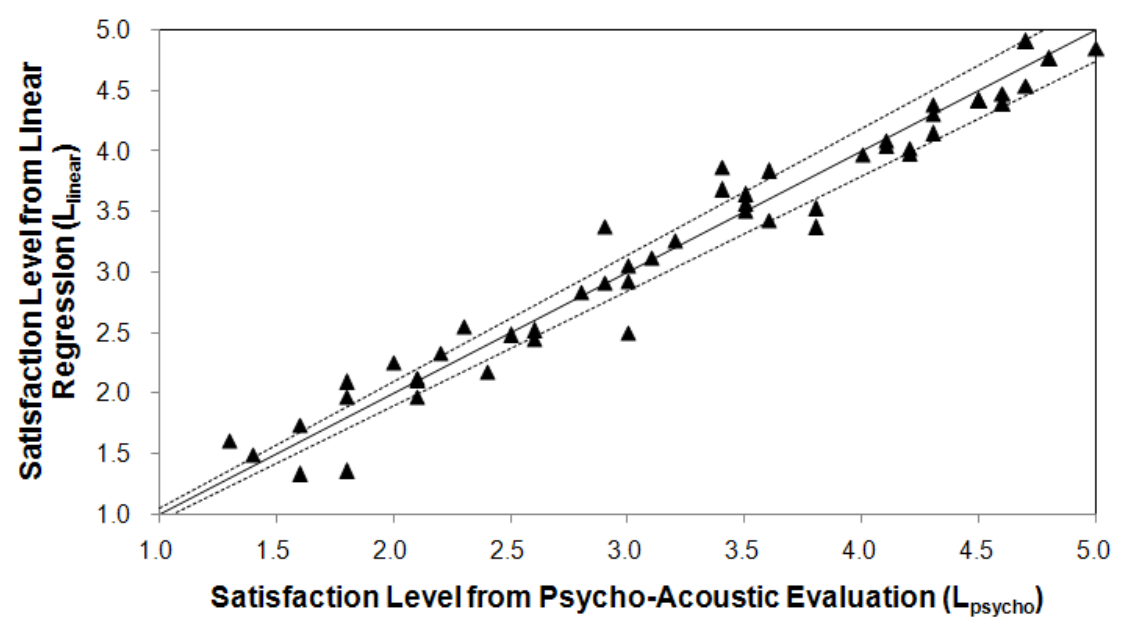

(a) Loudness.

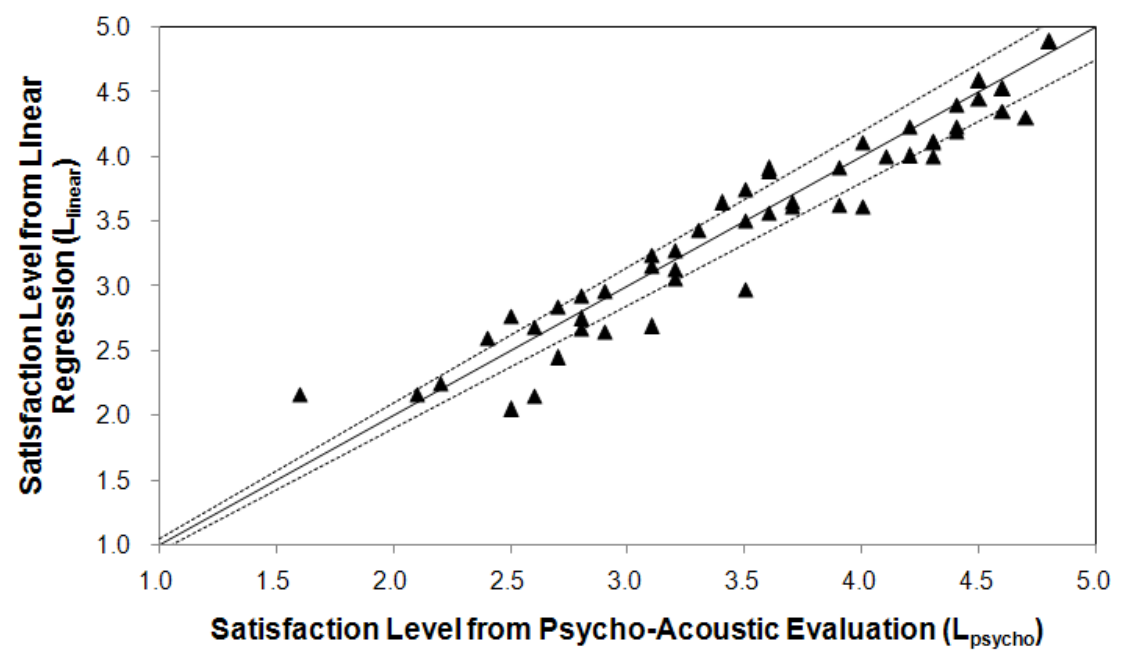

(b) Annoyance.

Fig. 2 Comparison between "Loudness" and "Annoyance" levels from the psycho-acoustic evaluation and from the linear regression as given in Eq. (1) (hidden line: $\pm 5 \%$ for the $L_{\text {linear }} / L_{\text {psycho }}$ curve).

\section{Back-propagation neural network}

In order to reflect non-linear characteristics for the human response to indoor noise, the authors applied the neural network algorithm to determine the respondents' logic for evaluating indoor noise. Rumelheart and McCelland (1986) developed the back-propagation neural network algorithm as the non-linear expansion of the least mean square algorithm. It uses the repetition rule of the differential calculus to learn the target information set. Through these learning processes, given input produces the required information as output.

The back-propagation neural network consists of three layers: the input layer, the hidden layer and the output layer. Each layer is connected by various nodes. Nodes in each layer form a network connected to one another by connectors of varying weight strength. When the input set is provided to the input nodes, these signals are translated by an activation function and output to nodes in the hidden layer. The signals input to the hidden layer are translated to the output layer in the same way. 
Comparing the target and the produced output yields the information necessary to modify the connecting weight strengths from the upper to the lower layer.

The back-propagation neural network algorithm thus can make a complete network by repeatedly modifying the connecting weight strength between nodes to minimize the error between the target (here, the observed survey results) and output produced by the neural network. The steps of the learning algorithm of the back-propagation neural network are set forth below.

(Step 1) Initialize the weight strength $\left(w_{j i}, w_{k j}\right)$ and offset $\left(\theta_{j}, \theta_{k}\right)$ between nodes for the neural network.

(Step 2) Set the group of information for learning.

(Step 3) Calculate the translated value from input nodes to each node in the hidden layer $\left(n e t_{p} j\right)$ and output value from the activation function $\left(o_{p j}\right)$ as given in Eqs. (8) (9).

$$
\begin{aligned}
n e t_{p j} & =\sum_{i} w_{j i} o_{p i}+\theta_{j} \\
o_{p j} & =f_{j}\left(n e t_{p j}\right)
\end{aligned}
$$

(Step 4) Calculate the translated value from nodes in the hidden layer to the connecting node in the output layer $\left(n e t_{p k}\right)$ and the output value from the activation function $\left(o_{p k}\right)$ as given in Eqs. (10) (11).

$$
\begin{gathered}
n e t_{p k}=\sum_{i} w_{k i} o_{p j}+\theta_{k} \\
o_{p k}=f_{k}\left(\text { net }_{p k}\right)
\end{gathered}
$$

(Step 5) Calculate the errors $\left(\delta_{p k}\right)$ between the produced output and the expected value $\left(t_{p k}\right)$ as given in Eq. (12).

$$
\delta_{p k}=\left(t_{p k}-o_{p k}\right) f_{k}^{\prime}\left(n e t_{k}\right)=\left(t_{p k}-o_{p k}\right) o_{p k}\left(1-o_{p k}\right)
$$

(Step 6) Calculate the errors in the hidden layer $\left(\delta_{p j}\right)$ as given in Eq. (13).

$$
\delta_{p j}=f_{j}^{\prime}\left(\text { net }_{j}\right) \sum \delta_{p k} w_{k j}=\sum \delta_{p k} w_{k j} o_{p j}\left(1-o_{p j}\right)
$$

(Step 7) Modify the weight strength and offset for the output layer as given in Eqs. (14) (15).

$$
\begin{gathered}
w_{k j}=w_{k j}+\alpha \delta_{p k} o_{p j} \text { where } \alpha \text { is learning coefficient } \\
\theta_{k}=\theta_{k}+\beta \delta_{p k} \quad \text { where } \beta \text { is bias coefficient }
\end{gathered}
$$

(Step 8) Modify the weight strength and offset for the hidden layer as given in Eqs. (16) (17).

$$
\begin{gathered}
w_{j i}=w_{j i}+\alpha \delta_{p j} o_{p i} \\
\theta_{j}=\theta_{j}+\beta \delta_{p j}
\end{gathered}
$$


(Step 9) Learn the next training data.

(Step 10) Repeat the learning process from Step 2 to Step 9 until the error is under the limiting value.

The neural network algorithm is analogous to the process of human thought, but it can fail to converge onto a target and fall into the local minima points. The convergent ability also varies according to the initial conditions and the accuracy depends on the amount of training data. Since the neural network can be expected to represent sufficiently the human nonlinear response to indoor noise, the authors adopted the back-propagation neural network as the means to develop the evaluation index of the indoor noise in this research.

This paragraph demonstrates how the neural network is constructed. The various acoustic parameters describing a room are the inputs and the crew's satisfaction with the noise is the output. The neural network inputs are $d B A, \mathrm{NR}, \mathrm{NC}, \mathrm{RC}, \mathrm{PSIL}$, and loudness, which $o_{p i}$ in Eq. (8) can be represented as $o_{p i}=[d B A, N R, N C, R C, P S I L, \text { Loudness }]^{T}$, and the parameter for the neural network output is the crew's index of satisfaction with the noise such as "Loudness" and "Annoyance", which $o_{p k}$ in Eq. (11) can be represented as $o_{p k}=$ Loudness or Annoyance .

Fig. 3 shows the construction of the back-propagation neural network. The hidden layer consists of 50 nodes and the activation function is the sigmoid function given in Eq. (18).

$$
f_{j}\left(x_{j}\right)=\frac{1}{1+\exp \left(-\mathrm{a} x_{j}+b\right)}
$$

Here, $x_{j}$ is the input, $a$ is the positive scaling constant which determines the slope of the sigmoid function and $b$ is the bias which determines the shift to the $\mathrm{x}$-axis for the sigmoid function.

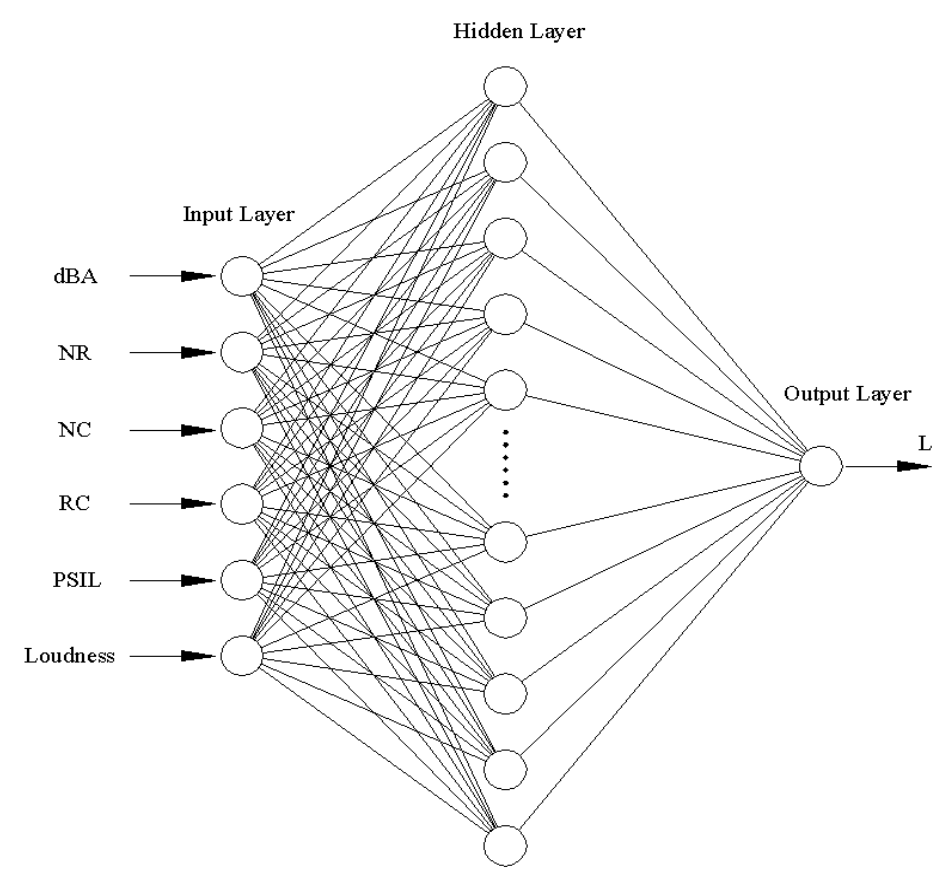

Fig. 3 Structure of the back-propagation neural network.

The data for learning is given from the psycho-acoustic evaluation in the previous chapter and parameters for input and output are normalized from 0 tol. Fig. 4 shows that the training data sets are learned by the back-propagation neural network within $\pm 5 \%$ for the data sets from psycho-acoustic evaluation in the previous chapter. Comparing results from multi-linear regression in the previous paragraph, it can be seen that the accuracy improves as shown in Fig. 4 for the given data sets. 


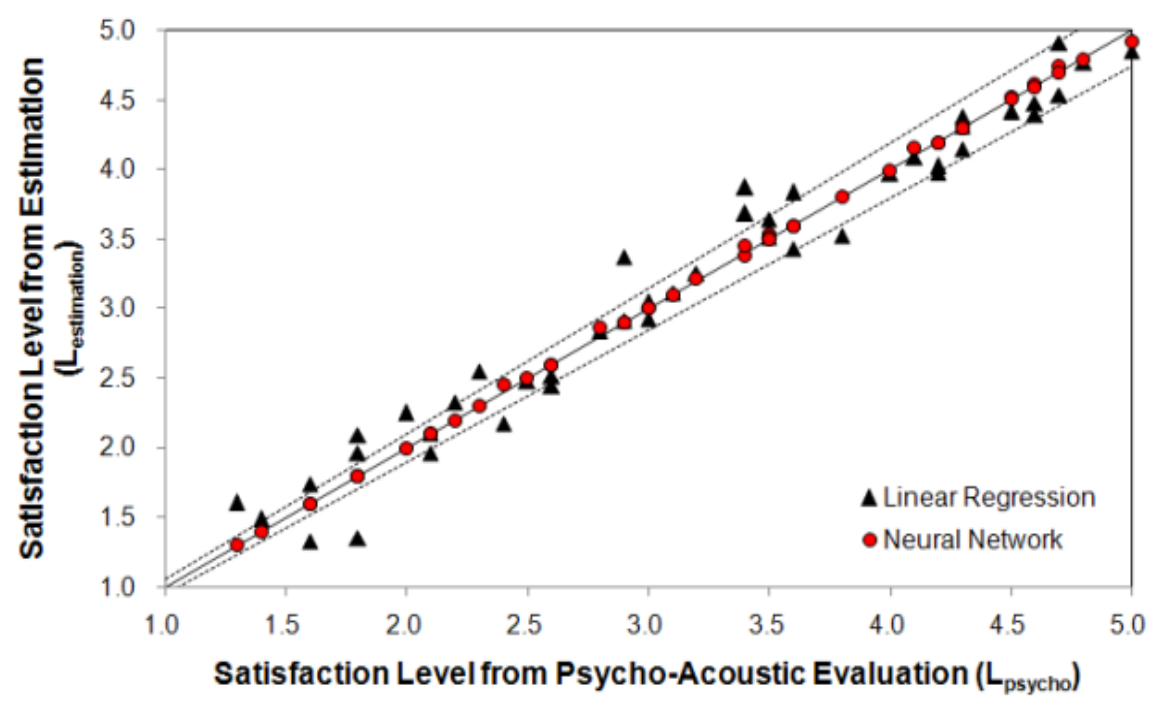

(a) Loudness.

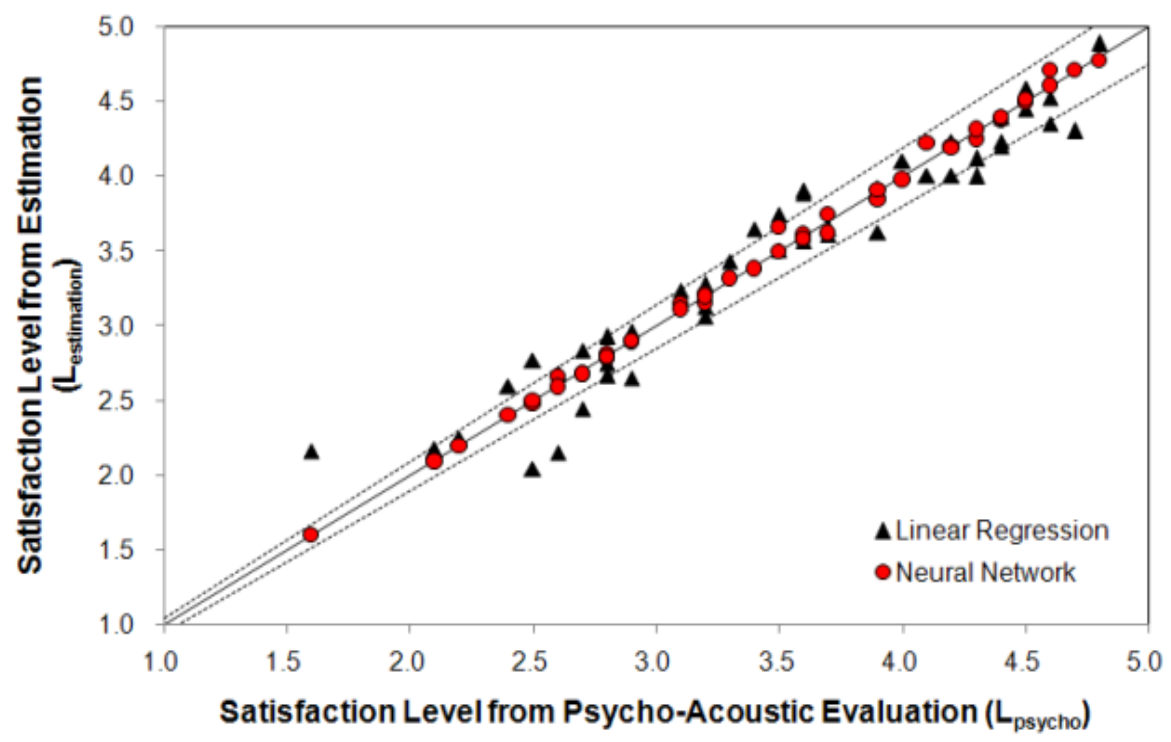

(b) Annoyance.

Fig. 4 Relation between results from the psycho-acoustic evaluation of the indoor noise, the results from multiple linear regressions, and results from a neural network (hidden line $=\mathrm{L}_{\text {psycho }} / \mathrm{L}_{\text {estimate }} \pm 5 \%$ ).

\section{VERIFICATION}

In this section, "Loudness" and "Annoyance" of the noise in 4 rooms, as is shown in Table 3, are evaluated using multiple regression and neural network models. These models were described in the previous section. The noise data in 4 rooms were recorded in the typical naval vessel separated to the learning data of the neural network in the previous section and evaluated by same jurors.

Fig. 5 shows the results for the psycho-acoustic evaluation of the indoor noise, the results from multiple linear regressions, and the results from a neural network. Fig. 5 shows that the estimation results from the neural network better coincide with those from the psycho-acoustic evaluation. When the learning process is performed with more sound data from the neural network, the accuracy of the estimation model of "Loudness" and "Annoyance" should be improved. 
Table 3 Noise data for 4 additionally measured rooms.

\begin{tabular}{|c|c|c|c|c|}
\hline \multirow{2}{*}{ Items } & \multicolumn{4}{|c|}{ Room No. } \\
\cline { 2 - 5 } & 1 & 2 & 3 & 4 \\
\hline$d B A$ & 62.39 & 59.54 & 55.08 & 51.27 \\
\hline NR & 57.40 & 54.50 & 50.20 & 46.20 \\
\hline NC & 61.80 & 58.20 & 52.00 & 46.20 \\
\hline RC & 57.40 & 54.50 & 50.20 & 47.30 \\
\hline PSIL & 54.54 & 51.64 & 47.22 & 43.69 \\
\hline Zwicker's Loudness [phon] & 80.40 & 77.40 & 73.10 & 70.20 \\
\hline "Loudness" & 4.10 & 3.80 & 3.00 & 2.10 \\
\hline "Annoyance" & 4.30 & 4.00 & 3.20 & 2.70 \\
\hline
\end{tabular}

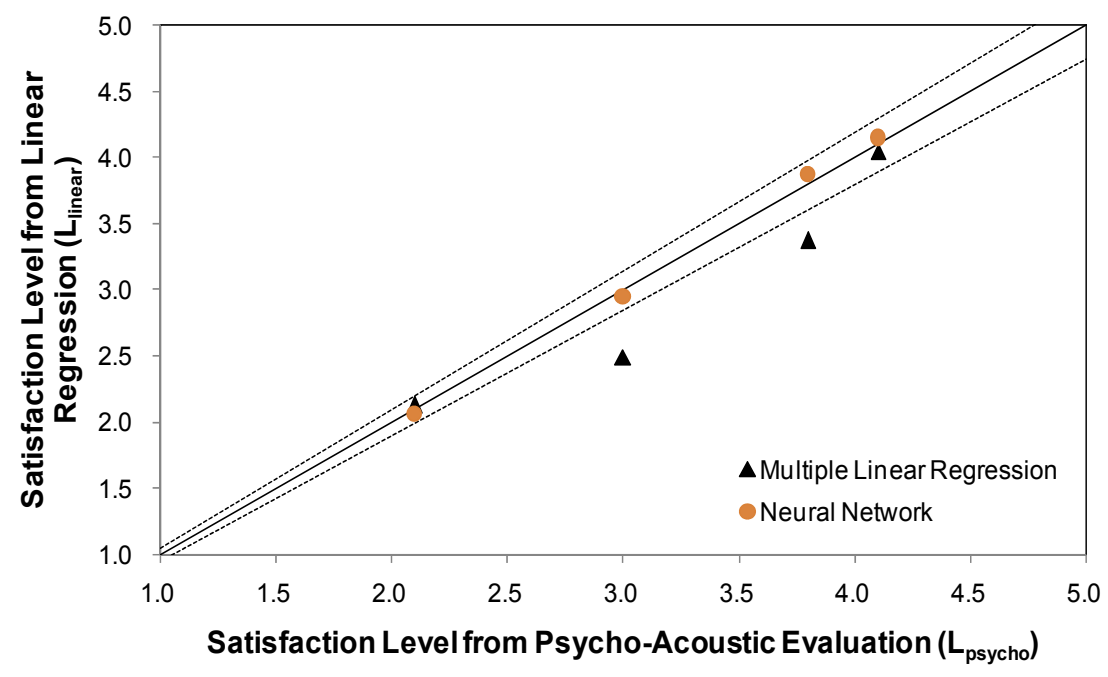

(a) Loudness.

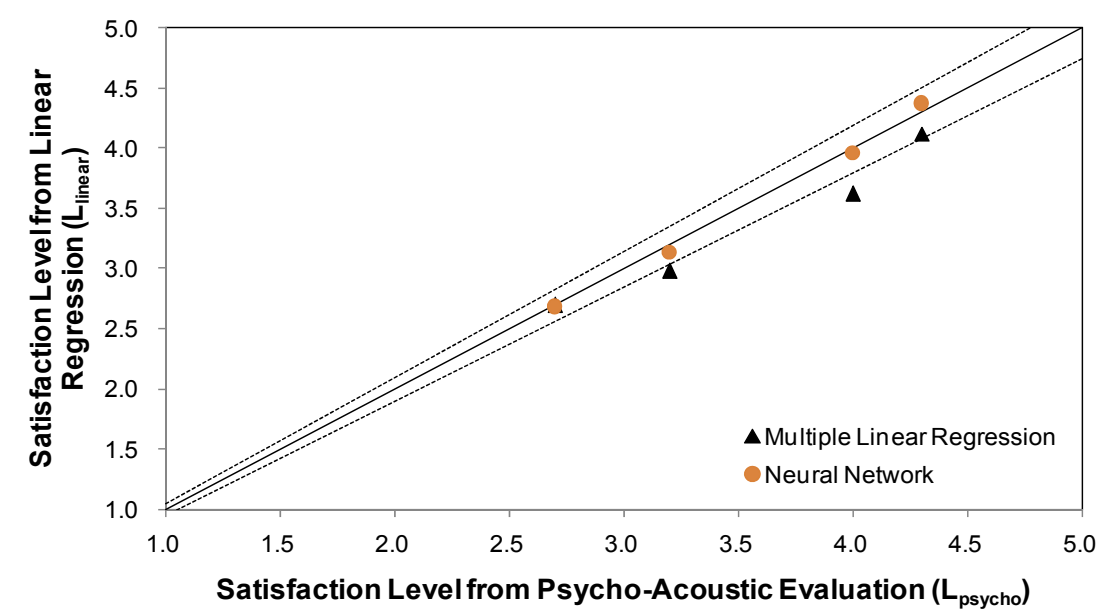

(b) Annoyance.

Fig. 5 Results for the psycho-acoustic evaluation for the indoor noise, the results from multiple linear regressions, and the results from a neural network (hidden line $=\mathrm{L}_{\text {psycho }} / \mathrm{L}_{\text {estimate }} \pm 5 \%$ ). 


\section{CONCLUSIONS}

The following conclusions are derived from the psycho-acoustic evaluation of noise inside a naval vessel.

(1) A noise estimation model in a naval vessel with "Loudness" and "Annoyance" level can be derived using a multiple regression analysis and neural network model using sound evaluation parameters, such as A-weighted sound pressure level, NR, NC, RC, PSIL, and Zwicker's Loudness.

(2) When the noise estimation models in a naval vessel derived from multiple regression analysis model is compared to that of a neural network model, the estimation from the neural network model is more coincidental than the results from the psychoacoustic evaluation when compared to the multiple regression analysis.

Since the psycho-acoustic evaluation is performed with finite audio tracks in this research, additional psycho-acoustic evaluation with additional audio tracks should be performed in order to determine a more generalized estimation and a neural network should be understood from these results. In addition, the variable parameters, such as the effect of background noise level and characteristics of various jurors, should be considered in order to get a more accurate estimation.

In this research, 6 psycho-acoustic parameters are used to evaluate "Loudness" and “Annoyance". However, we don't check the effectiveness of the each parameter to "Loudness" and "Annoyance" in this research. Therefore, factor analysis for these 6 parameters should be performed in the future. The results of this research should be very helpful in determining the noise limit inside a naval vessel.

\section{ACKNOWLEDGEMENTS}

This research was performed in the Defense Agency of Technology and Quality, and DTAQ verified that it did not contain any information related to military security.

\section{REFERENCES}

ANSI , 1977. ANSI S.3.14-1977(R886): Raring noise with respect to speech interference.

Beranek, L.L., 1957. Revised criteria for noise in buildings. Noise Control, 3, pp.19-27.

Blaizer, W.E., 1981. Revised noise criteria for design and rating of HVAC systems. ASHRAE Transactions, 87(1), pp.647657.

Blaizer, W.E., 1997. RC Mark-II: A refined procedure for rating the noise of Heating, Ventilation and Air-Conditioning (HVAC) systems in building. Noise Control Engineering Journal, 45(6), pp.243-250.

Chun, H.J., Kim, D.S., Ko, J.H. and Chang, S.I., 2008. Jury evaluation test for annoyance response of KTX(Korean Train Express) and ordinary train noise. Transactions of the Korean Society for Noise and Vibration Engineering, 18(3), pp. 335-344.

Han, H.S., Park, M.Y. and Cho, H.G., 2010. Study of the indoor noise limit for naval vessels considering the satisfaction of the crew. Journal of the Society of Naval Architects of Korea, 47(4), pp.589-597.

Hong, H.S., Shim, S.R. and Han, H.S., 2010. Psycho-acoustic evaluation and analysis of the indoor noise in cabins of a naval vessel to specify its allowable limit. Transactions of the Korean Society for Noise and Vibration Engineering, 21 (7), pp.650-656.

IMO, 1982. Noise Levels on Board Ships. IMO Publishing. International Organization for Standardization, 1975. ISO 5321975(E): Acoustic-method for calculating loudness level.

Joo, W.H. and Lee, T.K., 2009. Noise estimation and control for FPSO. Transactions of the Korean Society for Noise and Vibration Engineering, 19(8), pp.9-16.

Ju, D.H., Yun, J.H. and Kim, J.S., 2010. Establishing for regulation standards of architectural facility noise using psychoacoustic experiment. Transactions of the Korean Society for Noise and Vibration Engineering, 20(11), pp.1001-1008.

Kostan, C.W. and OS, G.J., 1962. Community reaction criteria for external noise. National Physical Laboratory Symposium. No.12. London: H.M.S.O. pp.373-387. 
Lee, S.K., Kim, T.G. and Lee, U., 2006. Sound quality evaluation based on artificial neural network. ICNC 2006, Part 1, LNCS, 4221, pp.545-554.

Lee, T.G., Ko, K.P., Kim, H.K., Song, G.G. and Kim, S.W., 2008. An experimental study on the subjective response for water supply and drain installations in apartment bathroom. Transactions of the Korean Society for Noise and Vibration Engineering, 18(6), pp.663-673.

Lee, P.J., Shim, M.H., Jeon, J.Y., 2010. Effects of different noise combinations on sleep, as assessed by a general questionnaire. Journal of Applied Acoustics, 71(9), pp.870-875.

Nannariello, J., Hodgson, M. and Fricke, F.R., 2001. Neural network prediction of speech levels in university classrooms. Applied Acoustics, 62(7), pp.749-767.

Remelheart, D.E. and McCelland, J.L., 1986. Parallel distributed processing 1. M.I.T. Press.

Tamura, Y., Kawada, T. and Sasazawa, Y., 1997. Effect of ship noise on sleep. Journal of Sound and Vibration, 205(4), pp. 417-425. 